\title{
Correcting second-order contamination in low-resolution spectra
}

\author{
V. Stanishev ${ }^{1 \star \star}$ vall@physto.se \\ Department of Physics, Stockholm University, Albanova University Center, 10691 Stockholm, Sweden
}

Received ; accepted

Key words techniques: spectroscopic - methods: data analysis - supernovae: individual: SN 2005cf - supernovae: individual: SN 2005hk

An empirical method for correcting low-resolution astronomical spectra for second-order contamination is presented. The method was developed for correcting spectra obtained with grism \#4 of the ALFOSC spectrograph at the Nordic Optical Telescope and the performance is demonstrated on spectra of two nearby bright Type Ia supernovae.

(c) 2007 WILEY-VCH Verlag GmbH \& Co. KGaA, Weinheim

\section{Introduction}

The availability of large format CCDs with high quantum efficiency over a wide wavelength range, makes possible most modern low-resolution spectrographs to have the capability to obtain spectra that cover the whole optical range (3200-10000A) in a single exposure. However, it follows from the theory of diffraction gratings (see, e.g.,Schroeder 2000) that different diffraction orders overlap, i.e. a photon with wavelength $\lambda^{m}$ in the $m$-th order will be diffracted at the same direction as a photon with wavelength $\lambda^{m+1}$ from the $m+1$-st order and thus both will be recorded at the same pixel on the detector. For diffraction gratings the relation between $\lambda^{m}$ and $\lambda^{m+1}$ is simple, $\lambda^{m}=(m+1) \lambda^{m+1} / m$ (but see Gutierrez-Moreno et al. (1994) for a case where this does not hold true due the a specific spectrograph design). Many spectrographs employ grisms instead of gratings, in which case the wavelength overlap relation is not that simple and is generally a non-linear function:

$$
\lambda^{m}=f^{(m+1 \rightarrow m)}\left(\lambda^{m+1}\right) .
$$

Most low-resolution astronomical spectra are obtained in the first diffraction order (with typical dispersion $\sim 2-$ $5 \AA$ pixel $^{-1}$ ) and thus beyond $\sim 6000 \AA$ will be contaminated by the blue light of the 2 nd-order, in many cases quite significantly. The order contamination greatly compromises the spectrophotometric accuracy but also affects the measured equivalent widths and profiles of the lines, as well as may introduce spurious lines when emission line objects are studied (Gutierrez-Moreno et al. 1994). The traditional way to overcome this problem has been to use blue light blocking filters or two different spectrometer settings. In either case one either loses the blue part of the spectrum or doubles the exposure time. Double-beam spectrographs are another solution, however, these are not as common instruments as the single-beam spectrographs. Besides, the dichroics used to split the beam may have non-uniform response at certain wavelengths, making the accurate flux calibration challenging. It would be therefore beneficial in many cases if a methodology for correcting single spectra for the higherorder contamination is available. However, little has been done in that direction so far. In previous works Bowers et al. (1997), Péroux et al. (2001) and Norman at al. (2002) only briefly outline the procedures they use to correct the order contamination in their spectra, and Gutierrez-Moreno et al. (1994), Leonard et al. (2002) and Steinmetz et al. (2006) discuss other aspects of the order contamination. I started working on the development of a method for 2 ndorder correction in early 2004 , largely motivated by the desire to optimize the Target-of-Opportunity observations of Type Ia Supernovae (SNe Ia) that began at the Nordic Optical Telescope (NOT) in the fall of 2003 using the ALFOSC spectrograph. Because grism \#4 (3200-9100 ̊) suffers of severe 2 nd-order contamination beyond $\sim 5800 \AA$, observations with the red grism \#5 (5000-9800 $\AA$; free of order contamination) were also obtained, but this doubled the observing time. Eventually, in the end of 2004 Szokoly et al. (2004) published a paper where they also presented in detail a method for correcting for the 2nd-order contamination; but my work was done independently, albeit arriving at quite a similar method.

\section{The method}

The observed spectrum in units of detected counts, $N\left(\lambda^{\mathrm{I}}\right)$, is $N\left(\lambda^{\mathrm{I}}\right)=N^{\mathrm{I}}\left(\lambda^{\mathrm{I}}\right)+N^{\mathrm{II}}\left(\lambda^{\mathrm{I}}\right)$, where $N^{\mathrm{I}}\left(\lambda^{\mathrm{I}}\right)$ is the true 1 st-order spectrum that would be detected if there was no contamination and $N^{\mathrm{II}}\left(\lambda^{\mathrm{I}}\right)$ is the 2 nd-order contamination, which is the true 2 nd-order spectrum $N^{\mathrm{II}}\left(\lambda^{\mathrm{II}}\right)$ mapped on the 1st-order wavelengths $\lambda^{\mathrm{I}}$ with Eq.1. The efficiency of a grism 11 at a given order should depend only on the parameters of the grim (Palmer \& Loewen 2006) 2 . In this

\footnotetext{
1 Throughout the paper I will refer to grisms, but the discussions are valid for diffraction grating as well.

2 The efficiency of reflection gratings depend sensitively on the polarization of the incidence light. However, the transmission gratings, to which class grisms belong, are practically free from the polarization effects
} 


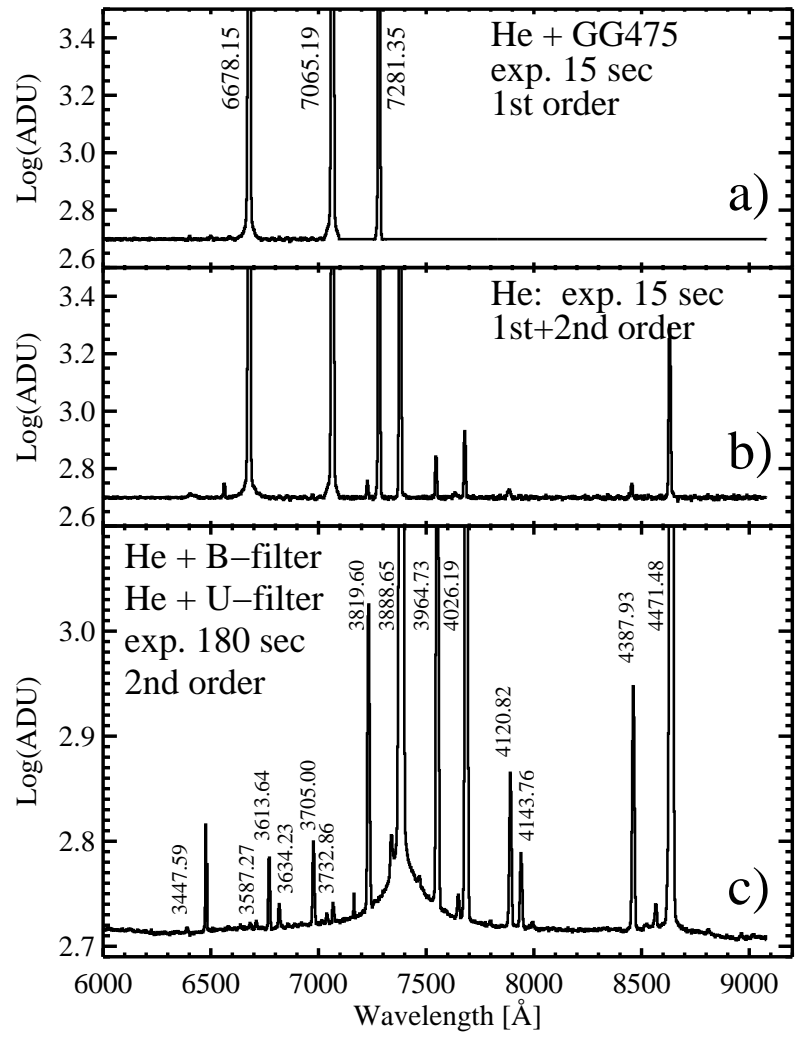

Fig. 1 He arc-lamp spectra a) with and b) without the blocking filter GG475. c) The combined spectrum obtained through the $U$ and $B$ filters. The lines used to obtain the wavelength solution for the second order are labeled in the c) panel. Some of these lines are clearly seen in the spectrum without the blocking filter.

case it is easy to show that for any object observed, the 2nd-order photons with wavelength $\lambda^{\mathrm{II}}$ that are recorded at wavelength $\lambda^{\mathrm{I}}$ in the first order, $N^{\mathrm{II}}\left(\lambda^{\mathrm{I}}\right)$, can be expressed as a function of the detected 1st-order photons $N^{\mathrm{I}}\left(\lambda^{\mathrm{I}}\right)$ :

$$
N^{\mathrm{II}}\left(\lambda^{\mathrm{I}}\right)=C\left(\lambda^{\mathrm{I}}\right) N^{\mathrm{I}}\left(\lambda^{\mathrm{I}}\right) \text {. }
$$

The function $C\left(\lambda^{\mathrm{I}}\right)$ is the ratio of the efficiencie 3 of the 2nd- and 1st-order $C\left(\lambda^{\mathrm{I}}\right)=E^{\mathrm{II}}\left(\lambda^{\mathrm{I}}\right) / E^{\mathrm{I}}\left(\lambda^{\mathrm{I}}\right)$, where $E^{\mathrm{II}}\left(\lambda^{\mathrm{I}}\right)$ is the efficiency of the 2nd-order again mapped on the 1st-order wavelengths. Thus the application of the method involves to steps: 1) wavelength transformation of the observed spectrum according to Eq.1, i.e. $N\left(\lambda^{\mathrm{I}}\right) \rightarrow$ $N\left(f^{(\mathrm{II} \rightarrow \mathrm{I})}\left(\lambda^{\mathrm{I}}\right)\right)$ and 2) multiplying $N\left(f^{(\mathrm{II} \rightarrow \mathrm{I})}\left(\lambda^{\mathrm{I}}\right)\right)$ by $C\left(\lambda^{\mathrm{I}}\right)$ and subtracting it from $N\left(\lambda^{\mathrm{I}}\right)$. What needs to be determined is the wavelength relation Eq.11 and $C\left(\lambda^{\mathrm{I}}\right)$.

The work on the method was first initiated by the observations of SN 2004S (Krisciunas et al. 2007) that were obtained at NOT in February 2004 using ALFOSC plus grism \#4. Because of the $\sim-31^{\circ}$ declination, the observations at La Palma were performed at very high airmass,

(Palmer \& Loewen 2006). Besides, in most cases the observed objects will not be strongly polarized.

3 efficiency here means the fraction of the incident photons in the corresponding order that are recorded on the detector.

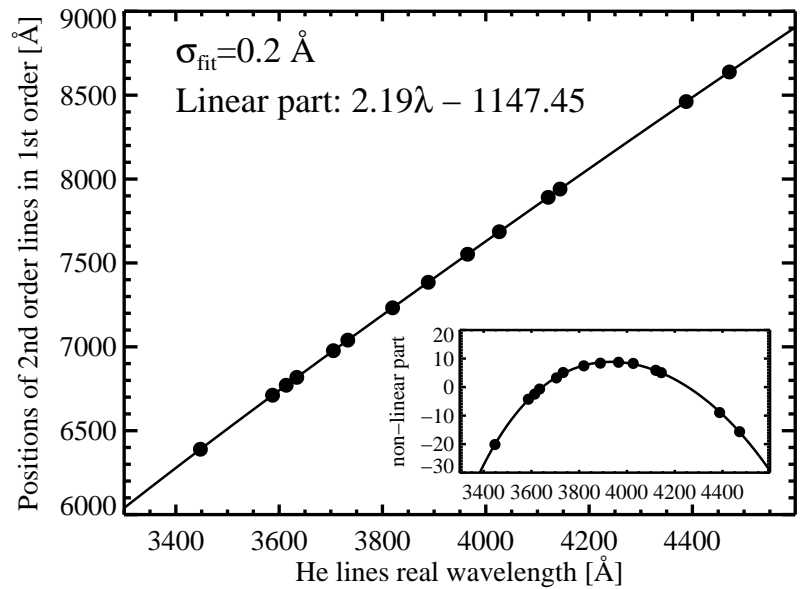

Fig. 2 The overlap relation between the 1 st and 2 nd order. The $1 \sigma$ scatter around the fit is $0.2 \AA$. Inset: the non-linear part of the fit.

often $X>2$, with slit along the parallactic angle to minimize the slit losses due to the differential atmospheric refraction (Filippenko 1982). At such a high airmass, the atmospheric refraction is large and the atmosphere effectively acts as a cross-disperser, similar to cross-dispersers in the Echelle spectrographs. This allowed on one occasion the 2nd-order to be spatially separated from the first order (see Fig.5. top), and the spectra at the two orders could be individually extracted. My first attempt was to use this observation to derive a method for correcting for the contamination. With too few blue photons detected (because of the high airmass) this was unsuccessful, but stimulated further work using observations of bright, blue stars with and without order-blocking filters in order to isolate the 2nd-order light. $N^{\mathrm{II}}\left(\lambda^{\mathrm{I}}\right)$ is the difference between the photon flux detected without and with the filter. Such observations were only obtained in November 2005. Using a slightly adjusted theoretical wavelength overlap function for grism \#4 provided by Per Rasmussen (Copenhagen Observatory, private communication) the method was successfully tested. However, more observations were obtained in May 2006, including dedicated observations to determine experimentally the wavelength overlap relation Eq.11. The results presented here are based on this later observing run.

To determine the overlap relation Eq.1 1 observations of $\mathrm{Ne}$ and He arc-lamps were obtained. The 1st-order wavelength solution was obtained from exposure of the $\mathrm{He}$ and $\mathrm{Ne}$ lamps simultaneously. Four 3 min long exposures of the He lamp without any filter and through the $U, B$, and the filter GG475 that block the light shortward of $\sim 4500 \AA$ were also obtained. The $U$ and $B$ filters served to block the 1 storder He lines, so that the faint 2 nd-order lines cold be measured to derive the wavelength solution for the 2nd-order (Fig.11). The other two exposures were only used to check if the filters introduced shifts to the line positions. Shifts of 1-2 pixels were found (and corrected) for the $U$ and $B$ filters, and none for GG475. Figure 2 shows the positions of 
the 2nd-order He lines as detected in the 1st-order vs. their real wavelengths. These data determine the order overlap relation and as can be seen from the inset in Fig.2 it is nonlinear. Note also that because of the higher dispersion, more lines could be detected in the second order than are usually seen in the first order.

Figure 3a shows the observations of the spectrophotometric standard Feige 66 with and without the GG475 filter. Figure $3 \mathrm{~b}$ shows the difference between the two spectra $\left(=N^{\mathrm{II}}\left(\lambda^{\mathrm{I}}\right)\right]^{4}$ and the wavelength transformed observed spectrum $N\left(\lambda^{\mathrm{I}}\right) \rightarrow N\left(f^{(\mathrm{II} \rightarrow \mathrm{I})}\left(\lambda^{\mathrm{I}}\right)\right)$. Note that the Hydrogen Balmer lines from the 2nd-order are clearly seen in the difference spectrum and their position are well matched by the lines in the transformed spectrum. Figure 3 shows the ratio $N^{\mathrm{II}}\left(\lambda^{\mathrm{I}}\right) / N\left(f^{(\mathrm{II} \rightarrow \mathrm{I})}\left(\lambda^{\mathrm{I}}\right)\right.$ which is the correction function $C\left(\lambda^{\mathrm{I}}\right)$. Because of the low sensitivity in the blue, the part between $5800 \AA$ and $6000 \AA$ is rather noisy and I fit a parametrized function (the thick line), which is the final correction function $C\left(\lambda^{\mathrm{I}}\right)$.

\section{Performance}

To demonstrate the performance of the algorithm I use observations of two Type Ia supernovae (SNe) 2005cf (Garavini et al. 2007) and 2005hk (Stanishev et al. 2007). Both SNe were observed at NOT with grisms \#4 and \#5 as a part of large observing campaigns involving many other telescopes. If there were no order contamination in grism \#4, the shape of the flux calibrated spectra with the two grisms would be the same. I therefore correct the grism \#4 spectra for the 2nd-order contamination and compare them with the ones obtained with grism \#5 in order to evaluate the performance of the algorithm. Note that the observing conditions were probably not perfectly photometric and so there is difference in the absolute flux level of the grism \#4 and \#5 spectra of the order of $\sim 5-15 \%$. Such small variations should not afect the relative flux calibration and are not problem for the analysis presented here.

Figure 4 presents the results for SN 2005cf. In Fig.4 4 a are shown the observed spectrum with grism \#4, the estimated correction and the corrected spectrum. Figure $4 \mathrm{~b}$ shows the uncorrected, flux calibrated spectra with the two grisms. Note that for this example neither the SN nor the spectrum of the spectrophotometric standard BD +33 2642 used for the flux calibration were corrected. Because BD +33 2642 is actually bluer than the SN, effectively flux is subtracted from and not added to the SN spectrum. After correcting for the 2nd-order contamination, the spectra with the two grisms line up nicely (Fig.4k). Figure $4 \mathrm{~b}$ clearly demonstrates not only the problem with the accurate relative

\footnotetext{
4 The GG475 filter transmission decreases nearly linearly from $97 \%$ to $92 \%$ between $5800 \AA$ and $9000 \AA$. The flux observed through the blocking filter was corrected accordingly. This correction was not in the original method and was included later after a note in Szokoly et al. (2004) that the blue light blocking filter may also affect the red part of the spectrum. This is the only influence that the paper of Szokoly et al. had on the work presented here.
}

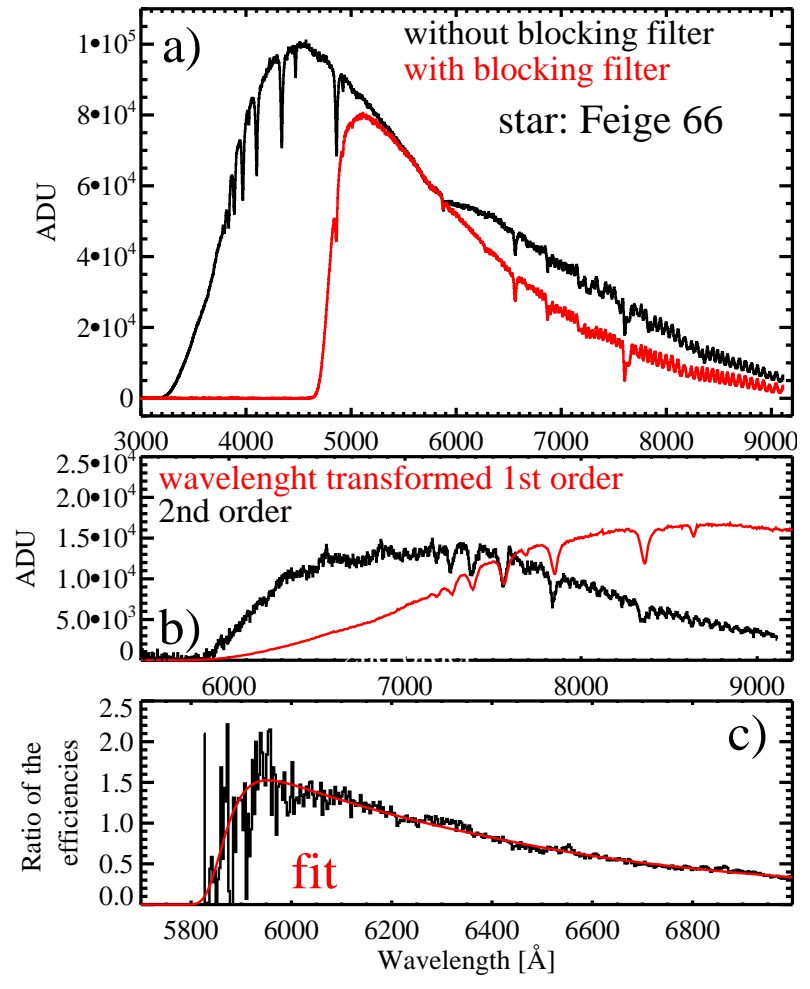

Fig. 3 a) The spectra of the hot sdO star Feige 66 with and without the GG475 blocking filter. b) The 2nd-order light and the wavelength transformed 1st-order light. c) The ratio between the 2 nd and 1 st-order light. The parametrized fit which is used for the 2nd-order contamination correction is also shown.

flux calibration, but also the negative effect on the measured line equivalent widths: in the uncorrected grism \#4 spectrum the strong Ca II infrared triplet at $\sim 8000 \AA$ is much weaker than in the grism \#5 spectrum.

With respect to SN 2005hk, I first correct the spectrophotometric standards to derive a contamination-free response function, which is then used to flux calibrate the SN spectra. On 2005 October 31 SN 2005hk was observed at high airmass and similarly to the case of the SN 2004S observations described in the previous section, the second order can be seen spatially well separated from the first order (Fig.5 top). The three ticks in Fig.5 (top) roughly correspond to wavelengths of $\sim 6700,7900$, and $9000 \AA$ from left to right. Cuts along the spatial axis were taken at these three positions in order to measure the FWHM of the spectral traces and the separation between them. The separations are 12, 10 and 7 pixels from blue to red wavelengths ( 1 pixel $=0.19$ arcsec). The FWHM of the 1st-order trace is 4.6 pixles at all cuts, but the second order trace FWHM decreases from 6 to 5 pixels from blue to red. The large separation between the orders allows to extract the 1st-order light with almost no 2nd-order contamination and to compare how the correction works not only with the grism \#5 spectrum but also with this 'clean' 1st-order extraction. I used an extraction aperture of 9 pixels width and thus be- 


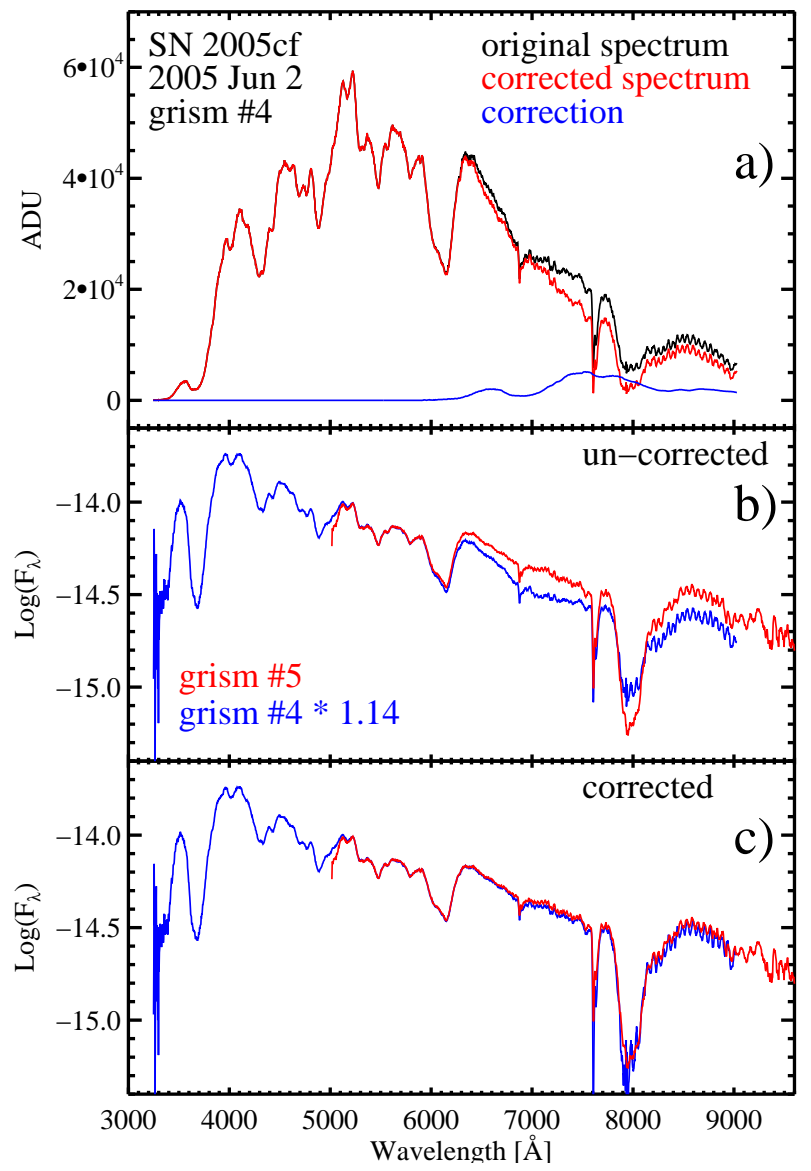

Fig. 4 a) The observed, the calculated correction and the corrected spectra of SN 2005cf observed at NOT on 2005 June 2.b) The flux calibrated uncorrected spectrum with grism \#4 compared with the spectrum with grism \#5. The effect of the 2 nd-order contamination in grism \#4 is very strong. c) Comparison of the two spectra after the one with grism \#4 has been corrected for the 2nd-order contamination. The two spectra match almost perfectly.

low $\sim 8000 \AA$ the extracted spectrum should be to a large extent contamination-free, while above $\sim 8000 \AA$ the contaminations will be somehow larger because the separation between the traces decreases to the red. Nonetheless, I find that the so extracted grism \#4 spectrum is in a good agreement with the grism \#5 one, indicating that nearly 'clean' extraction have been achieved. The results are presented in Fig. 5 and turning our attention to the 2nd-order contamination correction, I again find that the contamination has been very well corrected.

It should be noted that the experience from the observations shows that separation between the orders as large as 10 pixels is very rare. It is normally of the order of few pixels only and the two orders are blended togethet 15 . For the algorithm to work correctly, the spectrum that is to be corrected has to contain the entire light from both, the 1st and the 2nd order. Given the possibility for a significant separation be-

5 large seeing will cause blending even in case of large separations

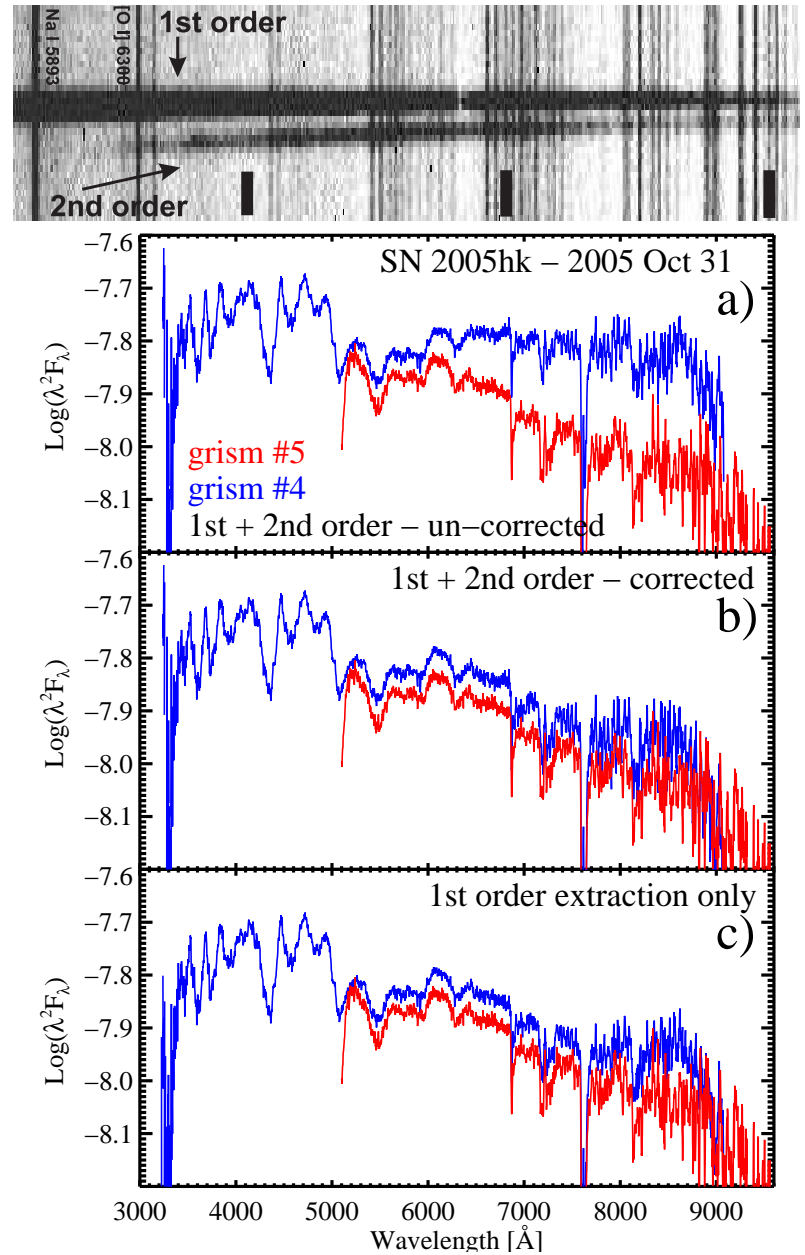

Fig. 5 Upper panel: The two-dimensional spectral image of SN 2005hk observed at NOT on 2005 October 31 with grism \#4. The 1st and 2 nd orders are clearly seen spatially separated (see the text for details). a) The flux calibrated uncorrected spectrum with grism \#4 compared with the spectrum with grism \#5. b) Comparison of the two spectra after the one with grism \#4 has been corrected for the 2nd-order contamination. c) Comparison between the grism \#5 spectrum with the uncorrected grism \#4 spectrum. In this case a narrow extraction aperture was used for grism \#4 spectrum, so that only the 1st order light was extracted.

tween the orders, to make sure that this is indeed the case one should carefully select the aperture position and width so that the light in both orders is fully extracted.

\section{Conclusions}

I present an empirical method for correcting low-resolution astronomical spectra for 2 nd-order contamination. The method was applied to grism \#4 of the ALFOSC instrument at the Nordic Optical Telescope, but can be used at any lowresolution grating/grism spectrograph. The performance of the method was tested on observations of two bright nearby Type Ia supernovae, SNe 2005cf and 2005hk, and in both 
cases the result was excellent, greatly improving the accuracy of the final flux calibrated spectra. Thus, in many situations the method would allow to reduce almost twice the observing time of programs that need to observe the whole optical range. Various Target-of-Opportunity programs on Supernovae, Gamma Ray Bursts and other transient object can greatly benefit from it. I expect the method to work very well for low-polarized objects with broad spectral features like SNe and GRBs. Its performance on objects with narrow spectral features and/or high polarization should be further investigated.

Acknowledgements. This work is supported in part by the European Community's Human Potential Program "The Physics of Type Ia Supernovae", under contract HPRN-CT-2002-00303. The author would like to thank the Göran Gustafsson Foundation for financial support. I am grateful to Jakob Jönson and Amanda Djupvik for performing the observation. ALFOSC is owned by the Instituto de Astrofisica de Andalucia (IAA) and operated at the Nordic Optical Telescope under agreement between IAA and the NBIfAFG of the Astronomical Observatory of Copenhagen.

\section{References}

Bowers, E. J. C., Meikle, W. P. S., Geballe, T. R., Walton, N. A., Pinto, P. A., Dhillon, V. S.: 1997, MNRAS 290, 663

Filippenko, A. V.: 1982, PASP 94, 715

Garavini, G., Nobili, S., S. Taubenberger, S., et al.: 2007, A\&A, accepted (astro-ph/0702569)

Gutierrez-Moreno, A., Heathcote, S., Moreno, H., Hamuy, M.: 1994, PASP 106, 1184

Leonard, D. C., Filippenko, A. V., Chornock, R., Foley, R. J.: 2002, PASP 114, 1333

Krisciunas, K., Garnavich, P. M., Stanishev, V., et al.: 2007, AJ 133,58

Norman, C., Hasinger, G., Giacconi, R., et al.: 2002, ApJ 571, 218

Palmer, Ch. \& Loewen, E.: 2005, Diffraction grating Handbook - 6th edition, Newport Corporation, available at http://gratings.newport.com/library/ handbook/handbook.pdf

Péroux, C., Storrie-Lombardi, L. J., McMahon, R. G., Irwin, M., Hook, I. M.: 2001, AJ 121, 1799

Schroeder, D.J.: 2000, Astronomical optics, 2nd ed.

Steinmetz, M., Zwitter, T., Siebert, A., et al.: 2006, AJ 132, 1645

Szokoly, G. P., Bergeron, J., Hasinger, G., et al.: 2004, ApJS 155, 271

Stanishev, V., et al.: 2007, in preparation 